\title{
Identification of a BAZ2A Bromodomain Hit Compound by Fragment Joining
}

Dalle Vedove, Andrea ; Cazzanelli, Giulia ; Corsi, Jessica ; Sedykh, Maria ; D’Agostino, Vito Giuseppe ; Caflisch, Amedeo ; Lolli, Graziano

\begin{abstract}
The bromodomains of BAZ2A and BAZ2B (bromodomain adjacent to zinc finger domain proteins 2) are among the most hard to drug of the 61 human bromodomains. While little is known about the role of BAZ2B, there is strong evidence for the opportunity of targeting BAZ2A in various cancers. Here, a benzimidazoletriazole fragment that binds to the BAZ2A acetyl lysine pocket was identified by a molecular docking campaign and validated by competitive binding assays and X-ray crystallography. Another ligand was observed in close proximity by soaking experiments using the BAZ2A bromodomain preincubated with the benzimidazole-triazole fragment. The crystal structure of BAZ2A with the two ligands was employed to design a few benzimidazoletriazole derivatives with increased affinity. We also present the engineering of a BAZ2A bromodomain mutant for consistent, high-resolution crystallographic studies.
\end{abstract}

DOI: https://doi.org/10.1021/acsbiomedchemau.1c00016

Posted at the Zurich Open Repository and Archive, University of Zurich

ZORA URL: https://doi.org/10.5167/uzh-212824

Journal Article

Published Version

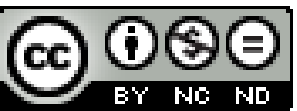

The following work is licensed under a Creative Commons: Attribution-NonCommercial-NoDerivatives 4.0 International (CC BY-NC-ND 4.0) License.

Originally published at:

Dalle Vedove, Andrea; Cazzanelli, Giulia; Corsi, Jessica; Sedykh, Maria; D’Agostino, Vito Giuseppe; Caflisch, Amedeo; Lolli, Graziano (2021). Identification of a BAZ2A Bromodomain Hit Compound by Fragment Joining. ACS Bio Med Chem Au, 1(1):5-10.

DOI: https://doi.org/10.1021/acsbiomedchemau.1c00016 


\title{
Identification of a BAZ2A Bromodomain Hit Compound by Fragment Joining
}

\author{
Andrea Dalle Vedove, ${ }^{\S}$ Giulia Cazzanelli, ${ }^{\S}$ Jessica Corsi, Maria Sedykh, Vito Giuseppe D’Agostino, \\ Amedeo Caflisch,* and Graziano Lolli*
}

Cite This: https://doi.org/10.1021/acsbiomedchemau.1c00016

Read Online

ACCESS | 씨 Metrics \& More | 回 Article Recommendations | SI Supporting Information

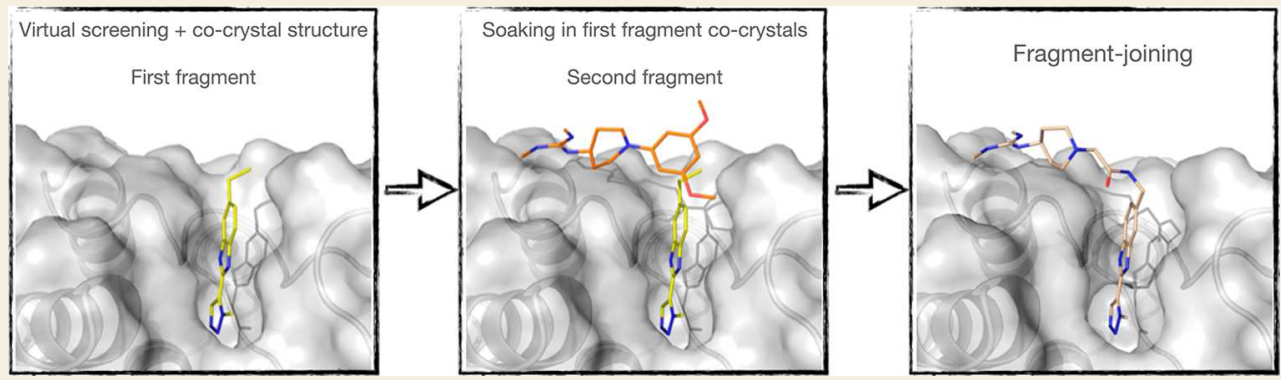

ABSTRACT: The bromodomains of BAZ2A and BAZ2B (bromodomain adjacent to zinc finger domain proteins 2) are among the most hard to drug of the 61 human bromodomains. While little is known about the role of BAZ2B, there is strong evidence for the opportunity of targeting BAZ2A in various cancers. Here, a benzimidazole-triazole fragment that binds to the BAZ2A acetyl lysine pocket was identified by a molecular docking campaign and validated by competitive binding assays and X-ray crystallography. Another ligand was observed in close proximity by soaking experiments using the BAZ2A bromodomain preincubated with the benzimidazole-triazole fragment. The crystal structure of BAZ2A with the two ligands was employed to design a few benzimidazole-triazole derivatives with increased affinity. We also present the engineering of a BAZ2A bromodomain mutant for consistent, high-resolution crystallographic studies.

KEYWORDS: bromodomains, BAZ2A, molecular docking, fragment screening, X-ray crystallography

AZ2A (bromodomain adjacent to zinc finger domain protein 2) is a large multidomain protein controlling rDNA accessibility and transcription through the nucleolar remodeling complex. Its bromodomain and PHD zinc finger domain recognize acetylated Lys 16 on histone $\mathrm{H} 4$, recruiting $\mathrm{HDAC} 1$ for heterochromatin formation. ${ }^{1}$

BAZ2A overexpression has been associated with prostate cancer aggressiveness, and its downregulation has been shown to impact the metastatic potential of prostate cancer cells. ${ }^{2}$ Chemical probes directed against its bromodomain also induced apoptosis in triple-negative breast cancer cells when used in combination with other bromodomain inhibitors. ${ }^{3}$ In this emerging picture, the inhibition of the BAZ2A bromodomain is better associated with the concomitant interference with a limited number of other bromodomains. Selective BAZ2A inhibitors should then not be disregarded in favor of exquisitely specific BAZ2A binders; instead, a narrow promiscuity among bromodomains could be advantageous for therapeutic purposes. The closest homologue of the BAZ2A bromodomain is its paralogue BAZ2B, whose function is still largely unknown.

BAZ2 bromodomains have a shallow acetylated lysine (Kac) binding pocket and are classified among the most difficult to target bromodomains. ${ }^{4}$ Only two chemical probes have been described so far, GSK2801 and BAZ2-ICR, binding BAZ2 bromodomains with affinities in the sub-micromolar range., Here, we describe the identification of a new BAZ2 bromodomain binding scaffold (compound 4) through fragment docking and its high-resolution structure in complex with an optimized, crystallization-prone BAZ2A mutant. An additional fragment was identified, which binds close to 4 , by soaking a limited number of compounds in BAZ2A/compound $\mathbf{4}$ cocrystals. By combining the two fragments, a BAZ2 hit compound was obtained that inhibits with an $\mathrm{IC}_{50}$ of $10 \mu \mathrm{M}$ the binding of BAZ2A to an acetylated peptide.

Wild-type BAZ2A (WT-BAZ2A) bromodomain crystallizes as very thin, often multiple, needles. Slightly thicker needles can be obtained by microseeding using fresh seeds. Seed concentration needs to be optimized for every batch to avoid

Received: May 25, 2021 
Table 1. Chemical Structures and Inhibitory Activities as Measured by AlphaScreen

\begin{tabular}{|c|c|c|c|c|c|c|}
\hline Compound & 2D structure & $\begin{array}{c}\text { IC }_{50} \text { BAZ2A } \\
(\mu \mathrm{M})\end{array}$ & $\mathbf{L} \mathbf{E}^{[\mathbf{a}]}$ & $\log P$ & $\begin{array}{c}\log D \\
\text { pH } 7.4\end{array}$ & $\operatorname{LLE}^{[\mathbf{b}]}$ \\
\hline 4 & & $28 \pm 7$ & 0.37 & 1.98 & 1.98 & 2.57 \\
\hline 17 & & $106 \pm 12$ & 0.31 & 2.57 & 2.57 & 1.40 \\
\hline 18 & & -- & -- & 2.16 & 1.08 & -- \\
\hline 19 & & $11 \pm 5$ & 0.22 & 0.21 & -2.23 & 4.75 \\
\hline 20 & & $14 \pm 8$ & 0.19 & 1.82 & -1.74 & 3.03 \\
\hline 21 & & $10 \pm 3$ & 0.26 & 1.64 & 1.64 & 3.36 \\
\hline
\end{tabular}

${ }^{a_{T}}$ The ligand efficiency (LE, $\mathrm{kcal} / \mathrm{mol}$ per heavy atom) is the measured binding free energy per non-hydrogen atom. ${ }^{b}$ The lipophilic ligand efficiency (LLE) is the difference between $\mathrm{pIC}_{50}$ and $\log \mathrm{P}$.

excessive nucleation. Diffraction from the needles obtained following this procedure is moderate and also affected by significant anisotropy.

A combination of surface entropy reduction (SER) and optimization of crystallographic contacts was applied to design a mutant with improved crystallization propensity. The SERp server (http://services.mbi.ucla.edu/SER/) ${ }^{7}$ and the "protein interfaces, surfaces and assemblies" service (PISA) at the European Bioinformatics Institute (http://www.ebi.ac.uk/ pdbe/prot_int/pistart.html $)^{8}$ were used for this purpose. The BAZ2A double mutant (BAZ2A-DM) E1845H/L1848S was produced, which crystallizes in two different forms: the usual trigonal form, yielding larger crystals isotropically diffracting to higher resolution than WT-BAZ2A, and a new monoclinic crystal form, which diffracts to $<1.5 \AA$. In both cases, the mutated residues are involved in favorable crystal contacts (Figure S1).

High-throughput docking was employed as the primary screening methodology. More in detail, the 54794 molecules of the Maybridge Screening Collection and Enamine Golden
Fragment libraries were docked with the program $\operatorname{SEED~}^{9,10}$ into the crystal structure of the BAZ2A bromodomain from a complex with a 4-propionylpyrrole derivative (5MGK). The structure of BAZ2A was kept rigid during docking and evaluation of binding energy. The binding energy in SEED is based on the CHARMM/CGenFF force field. ${ }^{11}$ The electrostatic effects of the solvent are treated in the continuum approximation by a numerical implementation ${ }^{12}$ of the generalized Born model. Fragment screening by SEED required $1-5 \mathrm{~s}$ per fragment depending on the number of pregenerated fragment conformers. SEED is available as an open source code from GitLab (https://gitlab.com/CaflischLab). The top 28 compounds (Figure S2) were used to define a library of 946296 compounds, extracted from the ZINC15 database by means of chemical similarity and substructure searches, which was used in a second docking cycle.

Among the 34 compounds prioritized by the computational screening (Figure S3), compounds $\mathbf{1 - 1 6}$ were purchased and tested by AlphaScreen on the BAZ2A bromodomain and its 

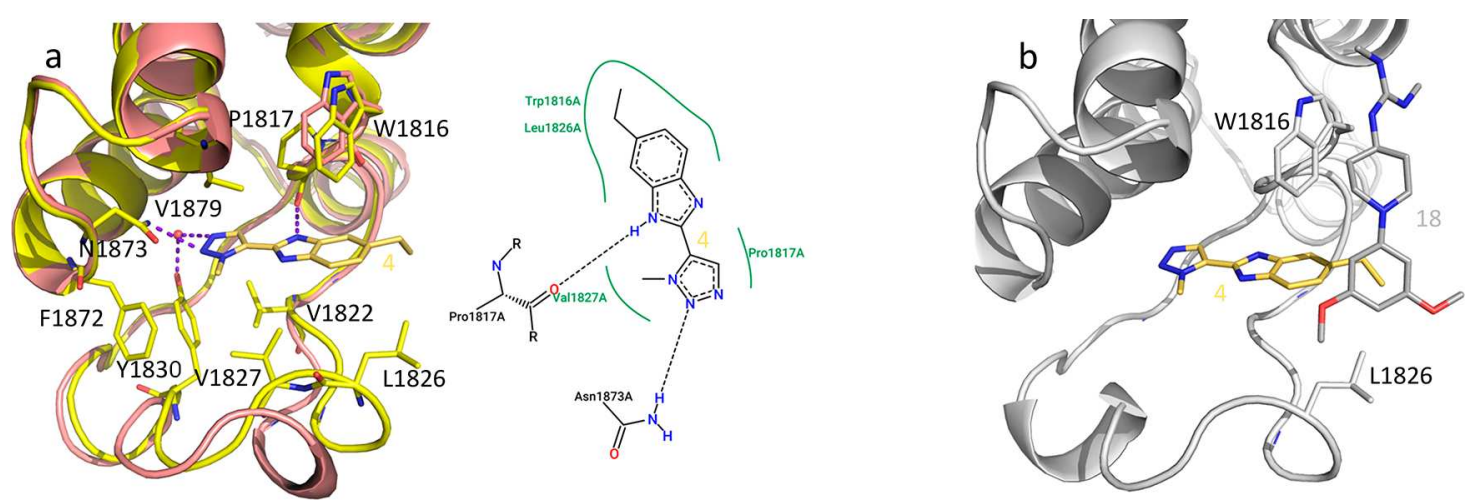

Figure 1. Binding of fragments to the BAZ2A bromodomain. (a) Compound 4 (yellow, PDB 7B7B) induces closure of the ZA loop; by comparison, the open ZA loop conformation is shown in dark pink. (b) Crystal structure of BAZ2A bromodomain in complex with both compounds 4 (yellow) and 18 (gray, PDB 7B7G).
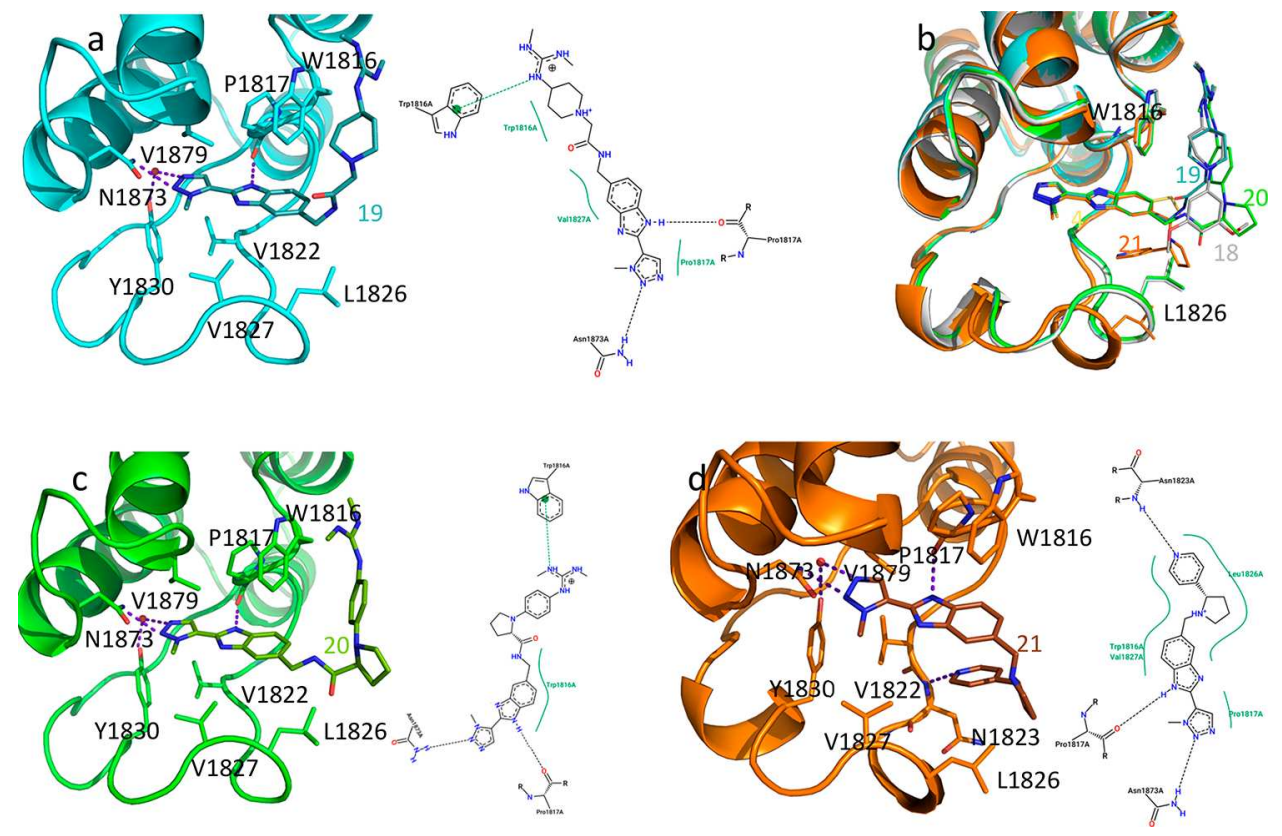

Figure 2. Fragment joining. (a) Compound 19 (PDB 7B7I) reproduces all interactions observed for $4+18$ (PDB 7B7G). (b) Superposition of all compounds: tail of compound 19 (cyan) almost perfectly superposes to 18 (gray). (c) Compound 20 (green, PDB 7B82) protrudes more from the bromodomain cavity. (d) Tail of Compound 21 (orange, PDB 7BC2) points in the opposite direction.

BAZ2B paralogue (Table S1). Only compound 4 showed a significant competition with the acetylated peptide for the bromodomain binding. The dose/response curve resulted in an $\mathrm{IC}_{50}$ of $28 \mu \mathrm{M}$ for the BAZ2A bromodomain (Table 1 and Figure S4).

Compound 4 was cocrystallized with the BAZ2A-DM bromodomain. Large three-dimensional polyhedral crystals were obtained, diffracting to $1.4 \AA$ resolution. Crystals belong to the $P 2_{1}$ space group, characterized by a crystallographic packing different from those observed before. Compound 4 binds in the BAZ2A Kac pocket (Figure 1a) by anchoring the N2 and N3 nitrogen atoms of its triazole headgroup to Asn 1873 and Tyr1830, respectively (this last interaction is water-mediated), reproducing the same hydrogen bonds observed for the carbonyl oxygen of the acetylated peptide (PDB 4QBM). A third hydrogen bond is formed by the benzimidazole nitrogen and the main chain oxygen of Pro1817 from the WPF shelf. Several hydrophobic and van der Waals interactions can be identified, with the most relevant involving Phe1872, the gatekeeper residue Val1879, and various residues from the ZA loop, namely,
Trp1816, Pro1817, Val1822, Leu1826, and Val1827. The experimentally observed binding mode reproduced the predicted docking pose, with minor deviations (Figure S5).

Notably, the Kac pocket reorganizes around compound 4 with the ZA loop folding on top of it. Leu1826 at the tip of the loop shifts by about $3 \AA$ (measured at $\mathrm{C} \alpha$ ) with respect to the deposited BAZ2A holo structures and is in contact with the benzimidazole ring of compound 4 (Figure 1a). On the other side, Trp 1816 also moves toward the benzimidazole ring, which is then squeezed between side chains of Leu1826 and Trp1816. Very similar flexibility of the ZA loop, adapting to the bound inhibitor, has been reported for various bromodomains, i.e., the paralogue BAZ2B. ${ }^{13}$ The introduced mutations $\mathrm{E} 1845 \mathrm{H}$ and L1848S do not interfere with the pocket structure nor with the ZA loop conformation being located away from both, neither do they alter the protein architecture in their vicinity. His 1845 and Ser1848 are involved in crystallographic interactions generating a denser packing not possible with the WT Glu1845 and Leu1848 (Figure S1c). 


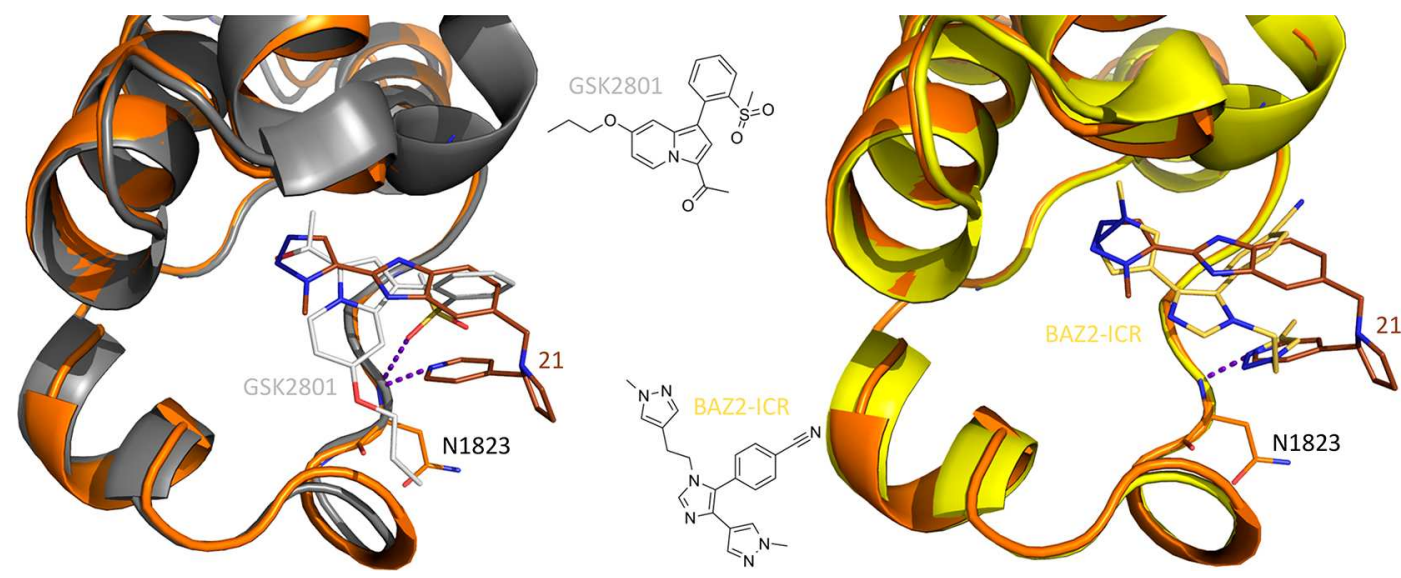

Figure 3. Binding mode of compound 21 and comparison with BAZ2 chemical probes. (Left) Compound 21 (brown, PDB 7BC2) forms a hydrogen bond with the backbone NH of Asn1823, as observed for GSK2801 (white, bound to BAZ2B in gray, PDB 4RVR). (Right) Compound 21 also shows an intramolecular ring stacking, as observed in the BAZ2-ICR analogue in complex with BAZ2B (yellow, PDB 4XUB).

The carbon atom in position 4 of the triazole ring only partially occupies the small cavity reserved for the methyl group of the Kac residue. To evaluate whether this cavity could be more advantageously explored, compound 17, carrying the smallest group in position 4 among the commercially available analogues of compound 4, was tested. 17 could establish a halogen bond via its bromine atom with Pro1817 carbonyl; alternatively, the triazole ring could rotate by $180^{\circ}$, placing the methyl group in the hydrophobic cavity. Compound 17 was, however, poorly active with an $\mathrm{IC}_{50}$ of $106 \mu \mathrm{M}$ (Table 1 and Figure S4).

We then took advantage of the nicely diffracting crystals obtained in complex with compound $\mathbf{4}$ for back-soaking a small library of 16 compounds that had shown some activity in the AlphaScreen assay. The back-soaking experiment (i.e., soaking of additional fragments after previous crystallization of the BAZ2A bromodomain with compound 4) is also useful for detecting the simultaneous binding of two or more fragments. Compound 18 bound close to 4, outside the Kac pocket (Figure 1b). The 1,3-dimethoxybenzene group is in contact with Leu1826 and is involved in a T-shaped stacking interaction with the benzimidazole ring of compound 4 . The piperidinyl dimethylguanidine moiety stacks parallel to Trp1816, being also engaged in a water-bridged hydrogen bond with Asp1813 carbonyl oxygen. Compound 18, binding at the interface between crystallographic copies, is stabilized by additional interactions with a symmetric BAZ2A chain, especially for the 1,3-dimethoxybenzene group.

Concurrent binding of compounds $\mathbf{4}$ and $\mathbf{1 8}$ was exploited to design molecules combining the $\mathbf{4}$ headgroup with tails reproducing the piperidinyl dimethylguanidine moiety of $\mathbf{1 8}$, either extensively (compounds 19 and 20) or partially (compound 21) (Table 1). The new compounds aim at adding to the 4 scaffold a cation $-\pi$ and/or a $\pi-\pi$ stacking interaction with Trp 1816 of either a guanidinium group or an aromatic ring. Compounds 19, 20, and 21 showed increased binding affinity with $\mathrm{IC}_{50}=11,14$, and $10 \mu \mathrm{M}$, respectively (Table 1 and Figure S4).

Compound 19 binds to BAZ2A largely superposing to the structure of $4+18$; the major difference is a $180^{\circ}$ rotation of the benzimidazole ring, which does not significantly affect its interactions with the WPF shell (Figure 2a,b). Its piperidinyl dimethylguanidine folds on top of Trp1816. The sensible increase in the observed interactions, with respect to 4 , is probably counterbalanced by the entropic penalty associated with the locked conformation.

Electron density for compound $\mathbf{2 0}$ is excellent for the triazole and benzimidazole ring, weaker for the prolinamide group (indicating multiple conformations), and progressively acquiring consistency for the 1,3-dimethyl-2-phenylguanidine region (Figure S6). Stacking with Trp1816 is poorer than in 19, with compound 20 retaining some conformational freedom and then paying a minor entropic cost. Overall, the prolinamide group excessively protrudes from the binding pocket, preventing optimal stacking of the phenylguanidine group with Trp1816 (Figure 2b,c).

Compound 21 deviates from the expected pose. Instead of pointing toward Trp1816, it orients its pyridine ring back in the pocket, forming a direct hydrogen bond to the main chain nitrogen of Asn 1823, also stacking with its own benzimidazole ring (Figure $2 b, d$ ). Only the trigonal crystal form was obtained, with the ZA loop in the open conformation, as the closed one, observed in monoclinic crystals, would clash with 21 . In the context of a more limited resolution $(2.0 \AA)$, the BAZ2A bromodomain selects the $S$-enantiomer that is, at least, largely predominant; geometric considerations also favor the observed enantioselectivity. Compound $\mathbf{2 1}$ recapitulates the hydrogen bond with Asn 1823 main chain nitrogen, explored by GSK2801 (and similar acetyl indole derivatives) and BAZ2-ICR in their complex with BAZ2B, for which the corresponding residue is Asn 1894 (Figure 3); ${ }^{5,6,14}$ it also formed an intramolecular $\pi-\pi$ stacking interaction, as observed in BAZ2-ICR (Figure 3). This is a more favorable interaction than the single $\pi-\pi$ stacking with the solvent-exposed Trp1816. Considering the observed enantioselectivity and the $\mathrm{IC}_{50}$ value of $10 \mu \mathrm{M}$ for the racemic mixture, the affinity for BAZ2A of the $21 S$-enantiomer is likely to be in the single-digit micromolar range.

Compounds 19-21 bind the BAZ2A bromodomain with higher affinity than 4. However, deterioration of the ligand efficiency (LE) occurs after fragment joining as a consequence of the weaker interactions with the rim of the binding site compared to those of the bottom of the Kac pocket. This can be rationalized considering that the rim consists mainly of solventexposed loops. Also, there is an entropic penalty due to the conformational constraints imposed on the ZA loop by the bound inhibitors, ${ }^{15}$ and the penalty might increase with the compound size. Growth of compound 4 was obtained through moieties of variable hydrophilicity, which importantly concur in 
defining $\log \mathrm{P}$ and $\log \mathrm{D}$ for 19-21 (Table 1 ). The lower LE of compound $\mathbf{2 1}$ with respect to that of $\mathbf{4}$ is compensated by a more favorable lipophilic ligand efficiency, in the context of optimal $\log \mathrm{P}$ and $\log \mathrm{D}$ values.

In conclusion, we have presented a screening strategy that combines docking and protein X-ray crystallography. Importantly, crystallography was used to validate the initial hit compound 4 obtained by docking and to provide suggestions for ligand growing. The protein structure-based hit expansion resulted in a 3-fold affinity increase for a difficult to target bromodomain. The potency of compound 21 could be further improved by substituting the methyl group on the triazole ring with a longer aliphatic tail retracing the path followed by the Kac side chain, as observed for GSK2801 (Figure 3).

\section{EXPERIMENTAL SECTION}

\section{Chemicals}

All compounds were obtained at a purity $>95 \%$, as determined by HPLC, with the exception of compound 19 (94\% pure). Compounds 1-18 were purchased from either Enamine Ltd. or SIA Chemspace. Compound 19 was obtained from SIA Chemspace, and compounds 20 and 21 were synthesized by Enamine Ltd.; NMR and LC/MS spectra are included in the Supporting Information for compounds 19-21.

\section{Molecular Docking}

In the first screening campaign, 54794 molecules were docked with the program SEED ${ }^{9,10}$ into the BAZ2A bromodomain (PDB 5MGK). A filter was subsequently applied with the following cutoffs on the SEED terms: HAC $>11$, vdW_eff $<-1.0 \mathrm{kcal} / \mathrm{mol}, \Delta$ Elec $<0.0 \mathrm{kcal} / \mathrm{mol}$ (HAC $=$ heavy atom count; vdW_eff $=$ van der Waals interaction energy/HAC; $\Delta \mathrm{Elec}=$ electrostatic $\overline{\text { difference }}$ upon fragment binding $=$ (electrostatic interaction in water - free energy of hydration of the fragment)). The 114 extracted compounds were filtered by visual inspection, by similarity to known bromodomain binders (Tanimoto coefficient set to 7.0), and by estimation of partial charge penalties (based on the level of analogy of a given compound with the internal training set of CGenFF). The 82 remaining molecules were rescored with ABSINTH, ${ }^{16}$ and only the 28 compounds with binding energy less than $-5 \mathrm{kcal} / \mathrm{mol}$ were selected (Figure S2).

Similarity and substructure searches were carried out against the ZINC15 database. The similarity search-Tanimoto coefficient and Morgan fingerprints of radius 1 -was parametrized to a similarity coefficient between 0.6 and 0.9. Anchor-like fragments (most buried moiety of the docked compounds involved in the conserved hydrogen bonds with Asn1873 and/or Tyr1830) were used for the substructure search by following the ALTA (anchor-base library tailoring) protocol. ${ }^{17}$ Similarity and substructure searches yielded 22412 and 966717 compounds, respectively. The obtained library was cleaned from PAINs, by means of a substructure search of the ZINC15 PAINs data set available on http://zinc15.docking.org/patterns/subsets/ pains/, from aggregators, using the list available on http://advisor. bkslab.org/faq/\#Data, and by duplicated compounds. Finally, the library was subjected to a last restricting step with the following rejection criteria: $\mathrm{MW}<-200 \mathrm{~g} / \mathrm{mol}, \log \mathrm{P}<-3$ or $>7$, HAC $<11$, absence of rings, number of rotatable bonds $>10$. Final library contained 946296 compounds.

The top compounds from the subsequent docking campaign were filtered with Tot eff (total binding energy $/ \mathrm{HAC}$ ) $<-0.74 \mathrm{kcal} / \mathrm{mol}$ and vdW_eff $<-0.91 \mathrm{kcal} / \mathrm{mol}$. The number of compounds was reduced to 130871 after filtering. Applied workflow for the final selection of compounds is depicted in Figure 5 of ref 10 and was here replicated. Structures of the final 34 molecules are shown in Figure S3, and their SEED energy values are reported in Table S2.

Compound 4 originated from the substructure search using the 1,2,3-triazole ring of Z802903370 (Figure S2).
Protein Production, AlphaScreen Assay, Crystallization, and Structure Solution

Mutations E1845H/L1848S were introduced in BAZ2AA-c002 plasmid (Addgene \#53623) through the QuikChange site-directed mutagenesis kit (Agilent) following the manufacturer's instructions. BAZ2A-DM was produced and crystallized similarly to WT-BAZ2A as previously described. ${ }^{18}$ BAZ2B bromodomain was obtained as detailed in an earlier work. ${ }^{19}$ Soaking of compounds in BAZ2A-DM/compound 4 crystals was performed in crystallization solution for $24-48 \mathrm{~h}$, in the absence of the acetyl-mimicking DMSO. ${ }^{20}$ Diffraction data were collected at the Elettra Synchrotron Light Source (Trieste, Italy), XRD1 and XRD2 beamlines. Data were processed, and structures were solved as described elsewhere. ${ }^{21}$ Data collection and refinement statistics are reported in Table S3. Electron densities $\left(2 F_{o}-F_{c}\right.$ and $F_{o}-$ $F_{c}$ polder OMIT map ${ }^{22}$ ) for the bound inhibitors are shown in Figure S6. AlphaScreen assay was conducted as detailed in ref 21 .

\section{ASSOCIATED CONTENT}

\section{Supporting Information}

The Supporting Information is available free of charge at https://pubs.acs.org/doi/10.1021/acsbiomedchemau.1c00016.

$2 \mathrm{D}$ structures of tested fragments and their binding competition activities on BAZ2A measured by AlphaScreen in single dose; SEED energy values for the 34 selected compounds; X-ray data collection and refinement statistics; BAZ2A crystallographic packings; $2 \mathrm{D}$ chemical structures of the 28 and 34 compounds selected in the first and second docking cycle, respectively; AlphaScreen dose-response curves for the selected compounds; comparison of experimental and docked poses for compound $4 ; 2 F_{\mathrm{o}}-F_{\mathrm{c}}$ and $F_{\mathrm{o}}-F_{\mathrm{c}}$ electron density maps for compounds whose structure in complex with the BAZ2A bromodomain; NMR and LC-MS spectra for compounds 19-21 (PDF)

PDB files for the docking sessions described in the article (ZIP)

\section{Accession Codes}

BAZ2A structures were deposited to the PDB with accession numbers 7B7B (compound 4), 7B7G (compound 4 + 18), 7B7I (compound 19), 7B82 (compound 20), and 7BC2 (compound 21). Atomic coordinates and experimental data will be released upon article publication.

\section{AUTHOR INFORMATION}

\section{Corresponding Authors}

Amedeo Caflisch - Department of Biochemistry, University of Zürich, CH-8057 Zürich, Switzerland; 이이.org/00000002-2317-6792; Email: caflisch@bioc.uzh.ch

Graziano Lolli - Department of Cellular, Computational and Integrative Biology - CIBio, University of Trento, 38123

Trento, Italy; 10 orcid.org/0000-0002-8536-5599; Email: graziano.lolli@unitn.it

Authors

Andrea Dalle Vedove - Department of Cellular, Computational and Integrative Biology - CIBio, University of Trento, 38123 Trento, Italy

Giulia Cazzanelli - Department of Cellular, Computational and Integrative Biology - CIBio, University of Trento, 38123 Trento, Italy 
Jessica Corsi - Department of Cellular, Computational and Integrative Biology - CIBio, University of Trento, 38123 Trento, Italy

Maria Sedykh - Department of Biochemistry, University of Zürich, CH-8057 Zürich, Switzerland

Vito Giuseppe D'Agostino - Department of Cellular, Computational and Integrative Biology - CIBio, University of Trento, 38123 Trento, Italy

Complete contact information is available at: https://pubs.acs.org/10.1021/acsbiomedchemau.1c00016

\section{Author Contributions}

${ }^{\S}$ A.D.V. and G.C. contributed equally to the work.

\section{Notes}

The authors declare no competing financial interest.

\section{ACKNOWLEDGMENTS}

We are grateful to the staff of XRD1 and XDR2 beamlines, Elettra Synchrotron Light Source (Trieste, Italy), for on-site assistance. Figures were prepared with PyMOL and PoseView. ${ }^{23}$ The research leading to these results has received funding from AIRC under MFAG 2017-ID. 19882 project, P.I. Lolli Graziano. A.C. is a recipient of an Excellence Grant [310030B-189363] of the Swiss National Science Foundation.

\section{REFERENCES}

(1) Zhou, Y.; Grummt, I. The PHD finger/bromodomain of NoRC interacts with acetylated histone $\mathrm{H} 4 \mathrm{~K} 16$ and is sufficient for rDNA silencing. Curr. Biol. 2005, 15, 1434-8.

(2) Gu, L.; Frommel, S. C.; Oakes, C. C.; Simon, R.; Grupp, K.; Gerig, C. Y.; Bär, D.; Robinson, M. D.; Baer, C.; Weiss, M.; Gu, Z.; Schapira, M.; Kuner, R.; Sültmann, H.; Provenzano, M.; Yaspo, M. L.; Brors, B.; Korbel, J.; Schlomm, T.; Sauter, G.; Eils, R.; Plass, C.; Santoro, R. BAZ2A (TIP5) is involved in epigenetic alterations in prostate cancer and its overexpression predicts disease recurrence. Nat. Genet. 2015, 47, $22-30$.

(3) Bevill, S. M.; Olivares-Quintero, J. F.; Sciaky, N.; Golitz, B. T.; Singh, D.; Beltran, A. S.; Rashid, N. U.; Stuhlmiller, T. J.; Hale, A.; Moorman, N. J.; Santos, C. M.; Angus, S. P.; Zawistowski, J. S.; Johnson, G. L. GSK2801, a BAZ2/BRD9 Bromodomain Inhibitor, Synergizes with BET Inhibitors to Induce Apoptosis in Triple-Negative Breast Cancer. Mol. Cancer Res. 2019, 17, 1503-18.

(4) Marchand, J. R.; Dalle Vedove, A.; Lolli, G.; Caflisch, A. Discovery of Inhibitors of Four Bromodomains by Fragment-Anchored Ligand Docking. J. Chem. Inf. Model. 2017, 57, 2584-2597.

(5) Chen, P.; Chaikuad, A.; Bamborough, P.; Bantscheff, M.; Bountra, C.; Chung, C. W.; Fedorov, O.; Grandi, P.; Jung, D.; Lesniak, R.; Lindon, M.; Müller, S.; Philpott, M.; Prinjha, R.; Rogers, C.; Selenski, C.; Tallant, C.; Werner, T.; Willson, T. M.; Knapp, S.; Drewry, D. H. Discovery and Characterization of GSK2801, a Selective Chemical Probe for the Bromodomains BAZ2A and BAZ2B. J. Med. Chem. 2016, 59, 1410-24.

(6) Drouin, L.; McGrath, S.; Vidler, L. R.; Chaikuad, A.; Monteiro, O.; Tallant, C.; Philpott, M.; Rogers, C.; Fedorov, O.; Liu, M.; Akhtar, W.; Hayes, A.; Raynaud, F.; Müller, S.; Knapp, S.; Hoelder, S. Structure enabled design of BAZ2-ICR, a chemical probe targeting the bromodomains of BAZ2A and BAZ2B. J. Med. Chem. 2015, 58, 2553-9.

(7) Goldschmidt, L.; Cooper, D. R.; Derewenda, Z. S.; Eisenberg, D. Toward rational protein crystallization: A Web server for the design of crystallizable protein variants. Protein Sci. 2007, 16, 1569-76.

(8) Krissinel, E.; Henrick, K. Inference of macromolecular assemblies from crystalline state. J. Mol. Biol. 2007, 372, 774-97.
(9) Majeux, N.; Scarsi, M.; Apostolakis, J.; Ehrhardt, C.; Caflisch, A. Exhaustive docking of molecular fragments with electrostatic solvation. Proteins: Struct., Funct., Genet. 1999, 37, 88-105.

(10) Goossens, K.; Wroblowski, B.; Langini, C.; van Vlijmen, H.; Caflisch, A.; De Winter, H. Assessment of the Fragment Docking Program SEED. J. Chem. Inf. Model. 2020, 60, 4881-93.

(11) Vanommeslaeghe, K.; Hatcher, E.; Acharya, C.; Kundu, S.; Zhong, S.; Shim, J.; Darian, E.; Guvench, O.; Lopes, P.; Vorobyov, I.; Mackerell, A. D., Jr. CHARMM general force field: A force field for drug-like molecules compatible with the CHARMM all-atom additive biological force fields. J. Comput. Chem. 2009, 31, 671-690.

(12) Scarsi, M.; Apostolakis, J.; Caflisch, A. Continuum Electrostatic Energies of Macromolecules in Aqueous Solutions. J. Phys. Chem. A 1997, 101, 8098-106.

(13) Marchand, J. R.; Lolli, G.; Caflisch, A. Derivatives of 3-Amino-2methylpyridine as BAZ2B Bromodomain Ligands: In Silico Discovery and in Crystallo Validation. J. Med. Chem. 2016, 59, 9919-27.

(14) Unzue, A.; Zhao, H.; Lolli, G.; Dong, J.; Zhu, J.; Zechner, M.; Dolbois, A.; Caflisch, A.; Nevado, C. The "Gatekeeper" Residue Influences the Mode of Binding of Acetyl Indoles to Bromodomains. J. Med. Chem. 2016, 59, 3087-97.

(15) Dolbois, A.; Batiste, L.; Wiedmer, L.; Dong, J.; Brütsch, M.; Huang, D.; Deerain, N. M.; Spiliotopoulos, D.; Cheng-Sánchez, I.; Laul, E.; Nevado, C.; Sledź, P.; Caflisch, A. Hitting a Moving Target: Simulation and Crystallography Study of ATAD2 Bromodomain Blockers. ACS Med. Chem. Lett. 2020, 11, 1573-80.

(16) Marchand, J. R.; Knehans, T.; Caflisch, A.; Vitalis, A. An ABSINTH-Based Protocol for Predicting Binding Affinities between Proteins and Small Molecules. J. Chem. Inf. Model. 2020, 60, 5188-202.

(17) Kolb, P.; Kipouros, C. B.; Huang, D.; Caflisch, A. Structure-based tailoring of compound libraries for high-throughput screening: discovery of novel EphB4 kinase inhibitors. Proteins: Struct., Funct., Genet. 2008, 73, 11-8.

(18) Spiliotopoulos, D.; Wamhoff, E. C.; Lolli, G.; Rademacher, C.; Caflisch, A. Discovery of BAZ2A bromodomain ligands. Eur. J. Med. Chem. 2017, 139, 564-72.

(19) Lolli, G.; Caflisch, A. High-Throughput Fragment Docking into the BAZ2B Bromodomain: Efficient in Silico Screening for X-Ray Crystallography. ACS Chem. Biol. 2016, 11, 800-7.

(20) Lolli, G.; Battistutta, R. Different orientations of low-molecularweight fragments in the binding pocket of a BRD4 bromodomain. Acta Crystallogr., Sect. D: Biol. Crystallogr. 2013, 69, 2161-4.

(21) Dalle Vedove, A.; Spiliotopoulos, D.; D’Agostino, V. G.; Marchand, J. R.; Unzue, A.; Nevado, C.; Lolli, G.; Caflisch, A. Structural Analysis of Small-Molecule Binding to the BAZ2A and BAZ2B Bromodomains. ChemMedChem 2018, 13, 1479-87.

(22) Liebschner, D.; Afonine, P. V.; Moriarty, N. W.; Poon, B. K.; Sobolev, O. V.; Terwilliger, T. C.; Adams, P.D. Polder maps: improving OMIT maps by excluding bulk solvent. Acta Crystallogr. D Struct. Biol. 2017, 73, 148-57.

(23) Stierand, K.; Rarey, M. Drawing the PDB: Protein-Ligand Complexes in Two Dimensions. ACS Med. Chem. Lett. 2010, 1, 540-5. 